\title{
Editorial
}

\section{Promoting Good Practice in Cognitive-Behavioural Psychotherapies}

The National Institute of Clinical Excellence (NICE) guidelines for anxiety (Panic and Generalized Anxiety Disorder) and depression were finally launched in December 2004, after much delay (see www.nice.org.uk). Given that these were such important documents, it might seem surprising that the launch went almost entirely unnoticed. Why was it surprising? Because the recommendations were ground breaking. CBT was identified as the first line treatment pretty much across the board, with huge resource implications. It is not the business of NICE to comment on resources (or the lack of these). However, it was noted in the anxiety guidelines that CBT should be available promptly from appropriately trained and supervised therapists. In many (if not most) areas, this strongly implies a huge change in emphasis in the delivery of psychological therapies. The (implicit) implications are staggering. With the honourable exception of Interpersonal Therapy in depression, other psychological therapies were not advocated - at all. In anxiety, combination treatment (medication and CBT) was not recommended as a first or second line treatment. Some medication (antipsychotic) was ruled out. Guided self-help (based on CBT principles) was "third choice". So the explicit recommendation is that good quality, empirically grounded psychotherapies (CBT, CBT and IPT in depression) should be widely and promptly available, whereas therapies without an empirical basis were not recommended.

So why the surprise at the lack of attention from the media? Think of a non-mental health area, say an empirically established surgical procedure. The technique known to be effective is clearly identified and recommended by NICE. The use of leeches is not advocated in the guidelines, but most practitioners are highly qualified and trained in the use of leeches. Advocates for leeching point out that the absence of evidence for the effectiveness of leeches does not mean that they are ineffective. Patients, they say, like being leeched. The suggestion that resources should be diverted from leeching to the (admittedly effective) surgical procedure is inappropriate because so many practise it. In any case, leeching treats the whole person rather than merely the symptoms of their disease. Well, maybe the person still has their symptoms at the end of leeching, but this doesn't matter too much as the real, underlying and difficult to define problem has been effectively dealt with, even if the patient doesn't know it.

So why the low profile of the NICE launch? The answer lies in the reasons for delaying the launch (originally due in the summer of 2004). The problem lay with the Medicines and Healthcare products Regulatory Agency (MHRA), which was urgently reviewing the potential adverse effects of SSRIs (withdrawal problems, impulsive violent acts and so on). As SSRIs featured prominently in the NICE guidelines, it was important to know what the MHRA recommendations might be. The media were understandably focused on this issue. The NICE 
guidelines groups did not get to see the MHRA recommendations until the last minute, and everything (two NICE guidelines and the MHRA report) was launched on the same day. The MHRA guidelines said, in essence, "SSRIs are probably not too bad", which attracted most of the comment and headlines. In the fuss, the message from NICE "CBT should be used first (in preference to SSRIs), and by the way it is not really available in the way it should be" was comprehensively submerged under the "it's OK to prescribe SSRIs as long as you do it carefully and have considered alternatives". Now, if I were selling SSRIs, I'd be rubbing my hands over this unexpectedly helpful way of putting out this kind of information.

So what do we do? Well, we need to make sure that the implications of the NICE guidelines are properly drawn out for those who are responsible for providing care for people with depression and anxiety, and for the patients that they seek to help. We need to look carefully at the implications of the recommendations for our own practice and research. For example, the anxiety guidelines support the application of shared decision making, referring to strategies such as "evidence based patient choice" (Hope, 1997) as ways of ensuring that patients are helped to understand the treatment options available to them, the evidence underpinning the options, and to be involved in the choice of which they receive. There is a range of other issues highlighted by the guidelines, from screening to follow-up, which urgently need further research.

In response to these imperatives, the journal intends to promote and prioritize research relevant to the NICE guidelines and their recommendations. This will also apply to forthcoming guidelines later this year (Obsessive Compulsive Disorder and Post-Traumatic Stress Disorder) as well as previous ones (Schizophrenia). We hope that this will not only result in new submissions, but also encourage clinical researchers and researcher clinicians to undertake new research at all levels. This issue includes an Accelerated Publication article by Cornwall, Scott, Garland and Pollinger on this basis. I look forward to receiving more articles that will inform the changes signalled by the NICE guidelines.

Paul Salkovskis

\section{Reference}

Hope, T. (1997). Evidence-Based Patient Choice. London: King's Fund. 